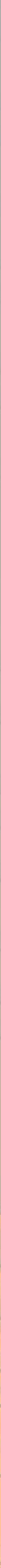




\title{
Maximal ideals and representations of twisted forms of algebras
}

\author{
Michael Lau and Arturo Pianzola
}

Given a central simple algebra $\mathfrak{g}$ and a Galois extension of base rings $S / R$, we show that the maximal ideals of twisted $S / R$-forms of the algebra of currents $\mathfrak{g}(R)$ are in natural bijection with the maximal ideals of $R$. When $\mathfrak{g}$ is a Lie algebra, we use this to give a complete classification of the finite-dimensional simple modules over twisted forms of $\mathfrak{g}(R)$.

\section{Introduction}

Let $S / R$ be a (finite) Galois extension of commutative, associative, and unital algebras over a field $k$, and let $\mathfrak{g}$ be a finite-dimensional central simple $k$-algebra. Let $\mathscr{L}$ be an $S / R$-form of $\mathfrak{g} \otimes_{k} R$, that is, an $R$-algebra $\mathscr{L}$ such that

$$
\mathscr{L} \otimes_{R} S \simeq \mathfrak{g} \otimes_{k} S
$$

as algebras over $S$.

In this paper we accomplish two tasks:

(1) We establish a natural correspondence between the maximal ideals of $\mathscr{L}$ and those of the base ring $R$.

(2) If $\mathfrak{g}$ is a Lie algebra, $k$ is algebraically closed of characteristic 0 , and $R$ is of finite type, we describe all the finite-dimensional irreducible modules of $\mathscr{L}$ and classify them up to isomorphism.

In what follows, we will denote $\mathfrak{g} \otimes_{k} S$ as $\mathfrak{g}(S)$. Recall that if $\Gamma$ is the Galois group of $S / R$, then there is a natural correspondence between the set of isomorphism classes of $S / R$-forms of $\mathfrak{g}(R)=\mathfrak{g} \otimes_{k} R$ and the pointed set of nonabelian Galois cohomology $\mathrm{H}^{1}\left(\Gamma\right.$, Aut $\left.{ }_{S \text {-alg }} \mathfrak{g}(S)\right)$. See [Knus and Ojanguren 1974], for example.

Funding from the Natural Sciences and Engineering Research Council of Canada is gratefully acknowledged.

MSC2010: primary 17B10; secondary 17B67, 12G05, 17A60.

Keywords: Galois descent, maximal ideals, finite-dimensional modules, multiloop algebras, twisted forms. 
For example, consider the multiloop algebra $\mathscr{L}(\mathfrak{g}, \sigma)$, where $\mathfrak{g}$ is a finite-dimensional Lie algebra over an algebraically closed field $k$ of characteristic 0 , and $\sigma$ is an $N$-tuple of commuting automorphisms

$$
\sigma_{1}, \ldots, \sigma_{N}: \mathfrak{g} \rightarrow \mathfrak{g}
$$

of finite orders $m_{1}, \ldots, m_{N}$, respectively. This is a $\mathbb{Z}^{N}$-graded Lie subalgebra of the Lie algebra $\mathfrak{g}(S)$, where $S=k\left[t_{1}^{ \pm 1}, \ldots, t_{N}^{ \pm 1}\right]$ :

$$
\mathscr{L}(\mathfrak{g} ; \sigma)=\bigoplus_{j \in \mathbb{Z}^{N}} \mathfrak{g}_{j} \otimes t_{1}^{j_{1}} t_{2}^{j_{2}} \cdots t_{N}^{j_{N}},
$$

where $\mathfrak{g}_{j}=\left\{x \in \mathfrak{g} \mid \sigma_{i}(x)=\xi_{i}^{j_{i}} x\right.$ for all $\left.i\right\}$, for fixed primitive $m_{i}$-th roots of unity $\xi_{i} \in k$. Then $\mathscr{L}(\mathfrak{g}, \sigma)$ is an $S / R$-form of $\mathfrak{g}(R)$, where $R=k\left[t_{1}^{ \pm m_{1}}, \ldots, t_{N}^{ \pm m_{N}}\right]$. The Galois group $\Gamma$ of $S / R$ is $\mathbb{Z}_{m_{1}} \times \cdots \times \mathbb{Z}_{m_{N}}$, and the corresponding (constant) 1cocycle in $\mathrm{H}^{1}\left(\Gamma\right.$, Aut $\left.{ }_{S \text {-alg }} \mathfrak{g}(S)\right)$ is the group homomorphism taking a fixed generator $\alpha_{i}$ of $\mathbb{Z}_{m_{i}}$ to $\sigma_{i}^{-1} \otimes 1$. Such algebras play an important role in affine Kac-Moody, toroidal, and extended affine Lie theory. ${ }^{1}$

We open the paper with a detailed investigation of the maximal ideals of twisted forms $\mathscr{L} .^{2}$ Given any ideal $\mathscr{I}$ of the $R$-algebra $\mathscr{L}$, we show that there is a unique $\Gamma$-stable ideal $J(\mathscr{Y}) \subseteq S$ for which $\mathscr{G} \otimes_{R} S$ maps to $\mathfrak{g} \otimes_{k} J(\mathscr{T})$ under the isomorphism $\mathscr{L} \otimes_{R} S \rightarrow \mathfrak{g} \otimes_{k} S$. As all maximal ideals $\mathscr{I}$ of the $k$-algebra $\mathscr{L}$ are $R$-stable, this produces a bijection $\psi: \mathscr{I} \mapsto J(\mathscr{I}) \cap R$ between maximal ideals of the $k$-algebra $\mathscr{L}$ and the set $\operatorname{Max}(R)$ of maximal ideals of $R$. Explicitly, $\psi^{-1}: I \mapsto I \mathscr{L}$ for maximal ideals $I \subseteq R$.

To have access to the attractive results of classical representation theory, we then assume that $\mathfrak{g}$ is a finite-dimensional simple Lie algebra and $R$ is of finite type over an algebraically closed field $k$ of characteristic 0 . The classification of finite-dimensional simple $\mathscr{L}$-modules $V$ proceeds by observing that the kernel of the representation $\phi: \mathscr{L} \rightarrow \operatorname{End}_{k}(V)$ is an intersection of a finite collection of distinct maximal ideals $\mathscr{I}_{1}, \ldots, \mathscr{I}_{n} \subseteq \mathscr{L}$. Given any maximal ideals $M_{1}, \ldots, M_{n} \in \operatorname{Max}(S)$ lying over the maximal ideals $\psi\left(\mathscr{I}_{1}\right), \ldots, \psi\left(\mathscr{I}_{n}\right) \in \operatorname{Max}(R)$, respectively, we obtain evaluation maps

$$
\mathrm{ev}_{M}: \mathscr{L} \hookrightarrow \mathfrak{g} \otimes_{k} S \rightarrow\left(\mathfrak{g} \otimes_{k} S / M_{1}\right) \oplus \cdots \oplus\left(\mathfrak{g} \otimes_{k} S / M_{n}\right) \simeq \mathfrak{g}^{\oplus n} .
$$

We then use properties of forms to show that $\mathrm{ev}_{M}$ is surjective and descends to an isomorphism ev $_{M}: \mathscr{L} / \operatorname{ker} \phi \stackrel{\sim}{\longrightarrow} \mathfrak{g}^{\oplus n}$. The finite-dimensional simple $\mathscr{L}$-modules $V$

\footnotetext{
${ }^{1}$ For simplicity of notation, we use integral powers of the variables $t_{i}$, though fractional exponents are sometimes used to work with the absolute Galois group of the base ring $R$ or with twisted modules for vertex algebras.

${ }^{2}$ Throughout this paper, all ideals are assumed to be two-sided unless there is an explicit mention to the contrary.
} 
are thus pullbacks of tensor products of $\mathfrak{g}$-modules along $\mathrm{ev}_{M}$ :

$$
V \simeq V(\lambda, M)=V_{\lambda_{1}}\left(M_{1}\right) \otimes_{k} \cdots \otimes_{k} V_{\lambda_{n}}\left(M_{n}\right)
$$

for some nonzero dominant integral highest weights $\lambda_{1}, \ldots, \lambda_{n}$ of $\mathfrak{g}$ (relative to a triangular decomposition $\left.\mathfrak{g}=\mathfrak{n}_{-} \oplus \mathfrak{h} \oplus \mathfrak{n}_{+}\right)$and maximal ideals $M_{1}, \ldots, M_{n} \in \operatorname{Max}(S)$, where $V_{\lambda_{i}}\left(M_{i}\right)$ is the simple $\mathfrak{g}$-module of highest weight $\lambda_{i}$, viewed as an $\mathscr{L}$-module via the composition of maps

$$
\mathscr{L} \stackrel{\mathrm{ev}_{M_{i}}}{\longrightarrow} \mathfrak{g} \otimes_{k} S / M_{i} \simeq \mathfrak{g} \rightarrow \operatorname{End}\left(V_{\lambda_{i}}\right) .
$$

Two such representations $V(\lambda, M)=V_{\lambda_{1}}\left(M_{1}\right) \otimes_{k} \cdots \otimes_{k} V_{\lambda_{m}}\left(M_{m}\right)$ and $V(\mu, N)=$ $V_{\mu_{1}}\left(N_{1}\right) \otimes_{k} \cdots \otimes_{k} V_{\mu_{n}}\left(N_{n}\right)$ are isomorphic $(\mathscr{L} / \operatorname{ker} \phi)$-modules, and thus isomorphic $\mathscr{L}$-modules, if and only if their highest weights are equal, relative to the induced triangular decomposition

$$
\mathscr{L} / \operatorname{ker} \phi=\mathrm{ev}_{M}^{-1}\left(\mathfrak{n}_{-}^{\oplus n}\right) \oplus \mathrm{ev}_{M}^{-1}\left(\mathfrak{h}^{\oplus n}\right) \oplus \mathrm{ev}_{M}^{-1}\left(\mathfrak{n}_{+}^{\oplus n}\right) .
$$

The cohomological interpretation of forms leads to an action of the group $\Gamma$ on $P_{+} \times \operatorname{Max}(S)$, for which $V(\lambda, M) \simeq V(\mu, N)$ if and only if $m=n$ and

$$
\left(\lambda_{i}, M_{i}\right)={ }^{\gamma_{i}}\left(\mu_{i}, N_{i}\right)
$$

for some $\gamma_{1}, \ldots, \gamma_{n} \in \Gamma$. This classification (Proposition 3.7) is then described in terms of $\Gamma$-invariant functions from the maximal spectrum $\operatorname{Max}(S)$ to the set $P_{+}$ of dominant integral weights. This gives a constructive description (Theorem 3.9) of the moduli space of finite-dimensional simple $\mathscr{L}$-modules in terms of finitely supported $\Gamma$-invariant functions $\operatorname{Max}(S) \rightarrow P_{+}$.

One of our main motivations in the present paper was to generalize and provide more intuitive proofs of previous work on (twisted) loop and multiloop algebras. See [Lau 2010; Senesi 2010] for a summary of past work on this problem. However, the interpretation of isomorphism classes as spaces of $\Gamma$-equivariant maps used in past work does not generalize to our context of twisted forms. Instead, the $\Gamma$-equivariant functions had to be reinterpreted as $\Gamma$-invariant functions $\operatorname{Max}(S) \rightarrow P_{+}$. This turned out to be the correct perspective to include cases where there is no natural action of $\Gamma$ on the space $P_{+}^{\times}$of nonzero dominant integral weights. More significantly, with new proofs, we have eliminated all dependence on the $\mathbb{Z}^{N}$-grading of $\mathscr{L}(\mathfrak{g}, \sigma)$, a point that was crucial in the arguments of [Lau 2010]. This lets us apply our work to nongraded contexts, including a classification of modules for the mysterious Margaux algebras explained in Section 4.

Perhaps the most striking feature of the present work is its nearly complete independence from the particular $S / R$-form under consideration. The maximal 
ideals of any $S / R$-form $\mathscr{L}$ of $\mathfrak{g}(R)$ are in bijection with $\operatorname{Max}(R)$, and the finitedimensional simple $\mathscr{L}$-modules are evaluation modules enumerated by finitely supported $\Gamma$-invariant maps $\operatorname{Max}(S) \rightarrow P_{+}$. Indeed, the only place where the Galois cocycle (and hence the isomorphism class) of the $S / R$-form plays an explicit role is in the isomorphism criterion for $\mathscr{L}$-modules (Proposition 3.7). But in many interesting examples, even this condition vanishes, as we illustrate in Section 4.

Notation. Throughout this paper, $k$ will denote a field. We let $k^{\times}=k \backslash\{0\}$ and denote the set of nonnegative integers by $\mathbb{Z}_{+}$. The category of finitely generated unital commutative associative $k$-algebras will be denoted by $k$-alg, and we will write $\operatorname{Max}(S)$ for the maximal spectrum of each $S \in k$-alg.

\section{Twisted forms and their maximal ideals}

In this section, $k$ will denote an arbitrary field and $S / R$ will be a finite Galois extension in $k$-alg with Galois group $\Gamma$. Let $\mathfrak{g}$ be a finite-dimensional central simple algebra over $k$, and let $R \in k$-alg. We may view $\mathfrak{g}(R) \cong \mathfrak{g} \otimes_{k} R$ as an algebra over $R$ by base change, the multiplication given by $(x \otimes r)(y \otimes s)=x y \otimes r s$ (for each $x, y \in \mathfrak{g}$ and $r, s \in R$ ). As before, $\mathscr{L}$ will denote an $S / R$-form of $\mathfrak{g}(R)$. Any such $\mathscr{L}$ is obviously an algebra over $k$ by restriction of scalars, and we may thus speak of $k$-ideals and $R$-ideals of $\mathscr{L}$, namely the ideals of $\mathscr{L}$ viewed as an algebra over $k$ and over $R$, respectively. ${ }^{3}$ The goal of this section is to classify the maximal $k$-ideals of $\mathscr{L}$.

Since Galois extensions are faithfully flat, we have the following general facts. See [Matsumura 1989, Theorem 7.5], for instance.

Lemma 2.1. Let $I$ be an ideal of $R$, and let $M$ be an $R$-module.

(1) The canonical map

$$
M \rightarrow M \otimes_{R} S, \quad x \mapsto x \otimes 1
$$

is injective. In particular, $R$ can be identified with a $k$-subalgebra of $S$.

(2) After viewing $R$ inside of $S$ via (1), IS is an ideal of $S$ and $R \cap I S=I$.

Up to coboundary, we can associate a Galois 1-cocycle

$$
u=\left(u_{\gamma}\right)_{\gamma \in \Gamma} \in Z^{1}\left(\Gamma, \operatorname{Aut}_{S-\operatorname{alg}}(\mathfrak{g}(S))\right)
$$

to $\mathscr{L}$, such that $\mathscr{L} \simeq \mathscr{L}_{u}=\left\{z \in \mathfrak{g} \otimes_{k} S \mid u_{\gamma} \gamma_{z}=z\right.$ for all $\left.\gamma \in \Gamma\right\}$. We therefore can (and henceforth will) view $\mathscr{L}$ as an $R$-subalgebra of $\mathfrak{g}(S)=\mathfrak{g} \otimes_{k} S$. Note that the $S$-algebra isomorphism

$$
\mathscr{L} \otimes_{R} S \simeq \mathfrak{g}(R) \otimes_{R} S=\mathfrak{g}(S)
$$

\footnotetext{
${ }^{3}$ We remind the reader that the word ideal means two-sided ideal.
} 
may be realized as the multiplication map

$$
\mu: \mathscr{L} \otimes_{R} S \rightarrow \mathfrak{g}(S), \quad\left(\sum_{i} x_{i} \otimes s_{i}\right) \otimes s \mapsto \sum_{i} x_{i} \otimes s_{i} s
$$

for all $\sum_{i} x_{i} \otimes s_{i} \in \mathscr{L}$ and $s \in S$. This will allow us to associate an ideal of $S$ to every $R$-ideal of $\mathscr{L}$.

Lemma 2.3. Let $\mathscr{I}$ be an $R$-ideal of $\mathscr{L}$. Then $\mathscr{I} \otimes_{R} S$ is an $S$-ideal of $\mathscr{L} \otimes_{R} S$, and there is a unique ideal $J=J(\mathscr{I}) \subseteq S$ such that $\mathfrak{g} \otimes_{k} J=\mu\left(\mathscr{I} \otimes_{R} S\right)$.

Proof. Fix a $k$-basis $\left\{x_{1}, \ldots, x_{m}\right\}$ of $\mathfrak{g}$. Let $J=J(\mathscr{I})$ be the set of all $s \in S$ for which there exists $\sum_{i=1}^{m} x_{i} \otimes s_{i} \in \mu\left(\mathscr{I} \otimes_{R} S\right)$ such that $s=s_{i}$ for some $i$. By the definition of $J$, it is clear that $\mu\left(\mathscr{I} \otimes_{R} S\right) \subseteq \mathfrak{g} \otimes_{k} J$. Moreover, since $\mathfrak{g} \otimes 1 \subseteq \mathfrak{g} \otimes_{k} S$ is a finitedimensional central simple $k$-algebra, it follows from the Jacobson density theorem that $x_{i} \otimes s \in \mu\left(\Phi \otimes_{R} S\right)$ for all $s \in J$ and for all $i \leq m$. Thus $\mathfrak{g} \otimes_{k} J \subseteq \mu\left(\Phi \otimes_{R} S\right)$. The uniqueness of $J$ is clear since the tensor product $\mathfrak{g} \otimes_{k} J$ is being taken over a field $k$.

Proposition 2.4. Let $\Phi_{1}$ and $\Phi_{2}$ be R-ideals of $\mathscr{L}$. Then $J\left(\Phi_{1}\right) \subseteq J\left(\Phi_{2}\right)$ if and only if $\Phi_{1} \subseteq I_{2}$. In particular, the map $J:\{R$-ideals of $\mathscr{L}\} \rightarrow\{$ ideals of $S\}$ is injective.

Proof. Let $\mathscr{I}=\mathscr{I}_{1}+\mathscr{I}_{2}$. The restriction of the multiplication map

$$
\mu: \mathscr{L} \otimes_{R} S \rightarrow \mathfrak{g}(S)
$$

to $\mathscr{I} \otimes_{R} S$ gives an isomorphism $\mu_{\mathscr{I}}: \mathscr{I} \otimes_{R} S \rightarrow \mathfrak{g} \otimes_{k} J(\mathscr{I})$ with $J(\mathscr{I})=J\left(\mathscr{I}_{1}\right)+J\left(\mathscr{I}_{2}\right)$. By flatness of $S / R$,

$$
\left(\mathscr{I} / \mathscr{I}_{2}\right) \otimes_{R} S \simeq \frac{\mathscr{I} \otimes_{R} S}{\mathscr{I}_{2} \otimes_{R} S}
$$

as $S$-modules. The injection $\mu_{\mathscr{g}}$ restricts to an isomorphism

$$
\mathscr{I}_{2} \otimes_{R} S \rightarrow \mathfrak{g} \otimes_{k} J\left(\mathscr{I}_{2}\right),
$$

so we see that

$$
\frac{\mathscr{I} \otimes_{R} S}{I_{2} \otimes_{R} S} \simeq \frac{\mathfrak{g} \otimes_{k} J(\mathscr{I})}{\mathfrak{g} \otimes_{k} J\left(\mathscr{I}_{2}\right)}=\mathfrak{g} \otimes_{k}\left(J(\mathscr{I}) / J\left(\mathscr{I}_{2}\right)\right) .
$$

Thus $\left(\mathscr{I} / \mathscr{I}_{2}\right) \otimes_{R} S=0$ if and only if $\mathfrak{g} \otimes_{k}\left(J(\mathscr{I}) / J\left(\mathscr{I}_{2}\right)\right)=0$; then by faithful flatness, $\mathscr{I} / \mathscr{I}_{2}=0$ if and only if $J(\mathscr{I}) / J\left(\mathscr{I}_{2}\right)=0$. That is, $\mathscr{I}_{1} \subseteq \Phi_{2}$ if and only if $J\left(\mathscr{I}_{1}\right) \subseteq J\left(\mathscr{I}_{2}\right)$.

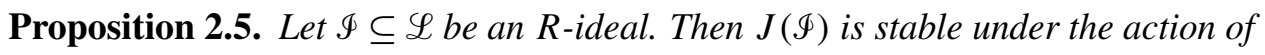
the Galois group $\Gamma=\operatorname{Gal}(S / R)$. 
Proof. As in the proof of Lemma 2.3, we fix a $k$-basis $\beta=\left\{x_{1}, \ldots, x_{m}\right\}$ of $\mathfrak{g}$. From the definition of $J=J(\mathscr{I})$, it is easy to see that $J$ is the ideal of $S$ generated by the set $E_{\beta}(\mathscr{I})$ of those elements $s \in S$ for which there is an element $\sum_{i} x_{i} \otimes s_{i} \in \mathscr{I}$ for which $s_{i}=s$ for some $i$. It is thus enough to show $\gamma_{s} \in J$ for all $\gamma \in \Gamma$ and $s \in E_{\beta}(\mathscr{Y})$.

Let $u \in Z^{1}\left(\Gamma\right.$, $\left.\operatorname{Aut}_{S \text {-alg }}(\mathfrak{g}(S))\right)$ be a cocycle corresponding to the $S / R$-form $\mathscr{L}$. Fix $\gamma \in \Gamma$, and write $u_{\gamma}\left(x_{i} \otimes 1\right)=\sum_{j=1}^{m} x_{j} \otimes a_{i j}$. Since $u_{\gamma}$ is an automorphism of $\mathfrak{g}(S)$, the matrix $A=\left(a_{i j}\right)$ is invertible in $M_{m}(S)$. Let $z=\sum x_{i} \otimes s_{i} \in \mathscr{I}$. It suffices to show that $\gamma_{s_{i}} \in J$ for $i=1, \ldots, m$. We have

$$
\begin{aligned}
\sum x_{i} \otimes s_{i} & =\mu(z \otimes 1)=\mu\left(u_{\gamma}^{\gamma} z \otimes 1\right)=\mu\left(\sum_{i} u_{\gamma}\left(x_{i} \otimes \gamma_{s_{i}}\right) \otimes 1\right) \\
& =\mu\left(\sum_{i} \gamma_{s_{i}} u_{\gamma}\left(x_{i} \otimes 1\right) \otimes 1\right)=\mu\left(\sum_{i} u_{\gamma}\left(x_{i} \otimes 1\right) \otimes \gamma_{s_{i}}\right) \\
& =\mu\left(\sum_{i, j} x_{j} \otimes a_{i j} \otimes \gamma_{s_{i}}\right)=\sum_{j} x_{j} \otimes\left(\sum_{i} a_{i j}{ }^{\gamma_{s}}\right) .
\end{aligned}
$$

In matrix form, we see that

$$
\left(\begin{array}{c}
\gamma_{S_{1}} \\
\vdots \\
\gamma_{s_{m}}
\end{array}\right)=\left(A^{t}\right)^{-1}\left(\begin{array}{c}
s_{1} \\
\vdots \\
s_{m}
\end{array}\right) .
$$

By definition, $s_{i} \in E_{\beta}(\mathscr{I}) \subseteq J$ for all $i$, and $\left(A^{t}\right)^{-1} \in M_{m}(S)$. Hence $\gamma_{s_{i}} \in J$ for all $i$.

Lemma 2.6. Let I be an ideal of $R$. Then $I \mathscr{L}$ is an ideal of $\mathscr{L}$, and $J(I \mathscr{L})=I S$.

Proof. It is obvious that $I \mathscr{L}$ is an ideal of $\mathscr{L}$. As $S$-modules (in fact, as $S$-algebras),

$$
I \mathscr{L} \otimes_{R} S=\mathscr{L} \otimes_{R} I S \simeq \mathscr{L} \otimes_{R} S \otimes_{S} I S \simeq \mathfrak{g} \otimes_{k} S \otimes_{S} I S \simeq \mathfrak{g} \otimes_{k} I S,
$$

so $J(I \mathscr{L})=I S$.

We now turn to the classification of maximal $k$-ideals $\mathscr{I}$ of the $S / R$-form $\mathscr{L}$.

Lemma 2.7. The sets of maximal $k$-ideals and maximal $R$-ideals of $\mathscr{L}$ coincide.

Proof. Let $\mathscr{I}$ be a maximal $k$-ideal of $\mathscr{L}$. We claim that $\mathscr{I}$ is stable under the action of $R$. For any $r \in R$, the space $r \mathscr{I}$ is clearly a $k$-ideal of $\mathscr{L}$, and if $r \mathscr{I} \nsubseteq \mathscr{I}$, then $\mathscr{I}+r \mathscr{I}=\mathscr{L}$ by the maximality of $\mathscr{I}$. The algebra $\mathscr{L}$ is perfect by descent considerations, as has already been noted in [Gille and Pianzola 2007], for instance. Thus

$$
\mathscr{L}=\mathscr{L} \mathscr{L}=(\mathscr{I}+r \mathscr{I}) \mathscr{L}=\mathscr{I} \mathscr{L}+\mathscr{I}(r \mathscr{L}) \subseteq \mathscr{I} \mathscr{L} \subseteq \mathscr{I},
$$


since $\mathscr{L}$ is an $R$-algebra. But this contradicts the proper inclusion $\mathscr{\subsetneq} \subsetneq \mathscr{L}$, so $r \Phi \subseteq \mathscr{I}$ as claimed. From this, it follows that every maximal $k$-ideal of $\mathscr{L}$ is also a maximal $R$-ideal of $\mathscr{L}$ and conversely.

Lemma 2.8. Let $M$ be a maximal ideal of $R$.

(1) There exist prime ideals of $S$ lying over $M$, and any such ideal is maximal. The group $\Gamma$ acts transitively on the set of such maximal ideals. In particular, this set is finite.

(2) $M S=\bigcap_{i} M_{i}$, where the intersection is taken over the (finite) set of maximal ideals of $S$ lying over $M$.

Proof. (1) This is well-known, but we recall the main ideas for completeness. From basic properties of Galois extensions, we know that $R=S^{\Gamma}$, and hence $S / R$ is integral. From this it follows that the set of prime ideals of $S$ lying over $M$ is not empty, that any such ideal is maximal, and that the action of $\Gamma$ on this set is transitive. (See [Bourbaki 1964, §2.1 Proposition 1 and \$2.2 Théorème 2].)

(2) Any maximal ideal $\mathfrak{m}$ of $S$ containing $M S$ will lie over $M$, since the intersection $\mathfrak{m} \cap R$ is a proper ideal of $R$ containing $M S \cap R$, which is equal to the maximal ideal $M$ by Lemma 2.1(2). Thus $\mathfrak{m}=M_{i}$ for some $i$, and $\bigcap_{i} M_{i}$ is the radical of $M S$. Since $S / R$ is flat,

$$
S / M S \simeq\left(R \otimes_{R} S\right) /\left(M \otimes_{R} S\right) \simeq(R / M) \otimes_{R} S .
$$

Let $L=R / M$, a field extension of $k$. Since the extension $S$ is Galois over $R$, general facts about base change guarantee that the extension $(R / M) \otimes_{R} S$ is Galois over $(R / M) \otimes_{R} R \simeq L$. (See [Milne 1980, §I.5], for instance.) That is, $S / M S$ is a Galois extension of $L$. Galois extensions are finite étale and the only such extensions of $L$ are products $L_{1} \times \cdots \times L_{m}$, where the $L_{i}$ are finite separable field extensions of $L$. We see from this that $S / M S$ has trivial Jacobson radical. Hence $M S$ is a radical ideal of $S$, and $M S=\bigcap_{i} M_{i}$.

Theorem 2.9. The map $\psi: I \mapsto I \mathscr{L}$ defines a bijection between the set of maximal ideals of $R$ and the set of maximal ideals of $\mathscr{L}$.

Proof. Let $\Phi$ be a maximal ideal of $\mathscr{L}$, and let $J=J(\mathscr{T}) \subseteq S$ be the ideal corresponding to $\mathscr{I}$. Let $P \subseteq S$ be a maximal ideal containing $J$, and let $M=P \cap R$. Since $S / R$ is integral, $M$ is a maximal ideal of $R$ [Bourbaki 1964, §2.1 Proposition 1].

As explained in Lemma 2.8(1), the Galois group $\Gamma$ acts transitively on the finite set $M_{1}, \ldots, M_{N}$ of maximal ideals $S$ lying over $M$. Since $J$ is $\Gamma$-stable (Proposition 2.5) and contained in a maximal ideal $P$ lying over $M$, we see that $J \subseteq \bigcap_{i=1}^{N} M_{i}$. By Lemma 2.8(2), $M S=\bigcap_{i=1}^{N} M_{i}$. Hence $J \subseteq M S$.

Note that $M \mathscr{L}$ is an ideal of $\mathscr{L}$ whose corresponding ideal is $M S$, by Lemma 2.6. By Proposition 2.4, $\mathscr{I} \subseteq M \mathscr{L}$. Since $M S=\bigcap_{i=1}^{N} M_{i}$ is a proper ideal of $S$, 
Lemma 2.3 guarantees that $M \mathscr{L}$ is a proper ideal of $\mathscr{L}$. Hence $\mathscr{I}=M \mathscr{L}$ by the maximality of $\mathscr{I}$, so the image of the map $\psi$ includes all maximal ideals of $\mathscr{L}$.

Let $I_{1}$ and $I_{2}$ be maximal ideals of $R$. If $I_{1} \mathscr{L}=I_{2} \mathscr{L}$, then $I_{1} S=I_{2} S$ by Proposition 2.4 and Lemma 2.6. Now Lemma 2.1(2) yields that $I_{1}=I_{2}$, hence that $\psi$ is injective. It remains only to check that $I \mathscr{L} \subseteq \mathscr{L}$ is maximal whenever $I \subseteq R$ is maximal. Suppose that $I \subseteq R$ is a maximal ideal, and let $\mathscr{I} \subseteq \mathscr{L}$ be a maximal ideal containing $I \mathscr{L}$. We have already shown that there is a maximal ideal $M \subseteq R$ for which $\mathscr{I}=M \mathscr{L}$. By Lemma 2.1(2) and Lemma 2.6,

$$
M=M S \cap R=J(M \mathscr{L}) \cap R=J(\mathscr{I}) \cap R .
$$

By Proposition 2.4, $J(I \mathscr{L}) \subseteq J(\mathscr{I})$, so

$$
I=I S \cap R=J(I \mathscr{L}) \cap R \subseteq J(\mathscr{I}) \cap R=M .
$$

By the maximality of $I$, we see that $I=M$. Hence $I \mathscr{L}=M \mathscr{L}=\mathscr{I}$ is a maximal ideal of $\mathscr{L}$.

As an application, we recover the following well-known fact; see [Knus and Ojanguren 1974, Corollary III.5.2].

Corollary 2.10. Let $A$ be an Azumaya algebra over R. Every (two-sided) maximal ideal of $\mathscr{A}$ is of the form IA for some maximal ideal I of $R$.

\section{Classification of simple modules}

We maintain the notation of the previous section but now assume that $\mathfrak{g}$ is a finitedimensional simple Lie algebra over an algebraically closed field $k$ of characteristic zero. The base ring $R$ will be of finite type in $k$-alg, and all modules (representations) will be of finite dimension over $k$. Unless explicitly indicated otherwise, $\otimes$ will denote a tensor product $\otimes_{k}$ taken over the base field $k$.

Let $\mathscr{L} \subset \mathfrak{g} \otimes S$ be an $S / R$-form of $\mathfrak{g}(R)$ as before, and let $\phi: \mathscr{L} \rightarrow \operatorname{End}_{k}(V)$ be a finite-dimensional irreducible representation of $\mathscr{L}$. We fix a cocycle $u \in$ $Z^{1}\left(\Gamma, \operatorname{Aut}_{S \text {-Lie }}(\mathfrak{g}(S))\right)$ so that $\mathscr{L}=\mathscr{L}_{u}$.

3a. Evaluation maps and simple modules. Since $\mathscr{L}$ is perfect, $\mathscr{L} / \operatorname{ker} \phi$ is a finitedimensional semisimple Lie algebra over $k$ [Lau 2010, Proposition 2.1]. Hence there is an isomorphism

$$
f: \mathscr{L} / \operatorname{ker} \phi \rightarrow \mathfrak{g}_{1} \oplus \cdots \oplus \mathfrak{g}_{n}
$$

for some collection of finite-dimensional simple $k$-Lie algebras $\mathfrak{g}_{1}, \ldots, \mathfrak{g}_{n}$. Let $\pi: \mathscr{L} \rightarrow \mathscr{L} / \operatorname{ker} \phi$ be the natural projection. Then

$$
\mathscr{L} / \operatorname{ker} \phi \simeq \mathscr{L} / M_{1} \oplus \cdots \oplus \mathscr{L} / \mathcal{M}_{n},
$$


where $M_{1}, \ldots, M_{n}$ are pairwise distinct maximal ideals of $\mathscr{L}$ whose intersection is $\operatorname{ker} \phi$. More precisely, we can take

$$
\mu_{i}=\pi^{-1} \circ f^{-1}\left(\mathfrak{g}_{1} \oplus \cdots \oplus \widehat{\mathfrak{g}_{i}} \oplus \cdots \oplus \mathfrak{g}_{n}\right)
$$

for $i=1, \ldots, n$, where $\widehat{\mathfrak{g}_{i}}$ indicates that the $i$-th summand is omitted. To classify the simple modules of $\mathscr{L}$, it thus suffices to consider quotients of $\mathscr{L}$ by maximal ideals. $^{4}$

Let $\mathscr{I} \subseteq \mathscr{L}$ be a maximal ideal. By Theorem 2.9, $\mathscr{I}=I \mathscr{L}$ for some maximal ideal $I \subseteq R$. Let $P \subseteq S$ be a maximal ideal lying over $I$, and let

$$
\epsilon: S \rightarrow S / P \simeq k
$$

be the natural evaluation map. ${ }^{5}$ Then the composition

$$
\operatorname{ev}_{P}: \mathscr{L} \hookrightarrow \mathfrak{g} \otimes S \stackrel{1 \otimes \epsilon}{\longrightarrow} \mathfrak{g} \otimes k \simeq \mathfrak{g}
$$

is a homomorphism of $k$-Lie algebras.

Proposition 3.3. The map $\operatorname{ev}_{P}: \mathscr{L} \rightarrow \mathfrak{g}$ is surjective and has kernel $\mathscr{I}=(P \cap R) \mathscr{L}$.

Proof. The multiplication map $\mu: \mathscr{L} \otimes_{R} S \rightarrow \mathfrak{g}(S)$ is an isomorphism (2.2), so given any element $x \in \mathfrak{g}$, there exist elements $z_{i} \in \mathscr{L}$ and $t_{i} \in S$ such that

$$
\mu\left(\sum_{i} z_{i} \otimes t_{i}\right)=x \otimes 1
$$

That is, if $z_{i}=\sum_{j} x_{j} \otimes s_{i j}$ for some $k$-basis $\left\{x_{j}\right\}$ of $\mathfrak{g}$ and $s_{i j} \in S$, then

$$
\sum_{i, j} x_{j} \otimes s_{i j} t_{i}=x \otimes 1
$$

Applying the map $1 \otimes \epsilon$ introduced in (3.1), we get $\sum_{i, j} x_{j} \otimes \epsilon\left(s_{i j}\right) \epsilon\left(t_{i}\right)=x \otimes 1$. But $\mathscr{L}$ is closed under multiplication by elements of $k$, so $\sum_{i} \epsilon\left(t_{i}\right) z_{i} \in \mathscr{L}$, and

$$
\operatorname{ev}_{P}\left(\sum_{i} \epsilon\left(t_{i}\right) z_{i}\right)=\sum_{i, j} x_{j} \epsilon\left(s_{i j}\right) \epsilon\left(t_{i}\right)=x
$$

Hence $\mathrm{ev}_{P}$ is surjective.

Let $z=\sum_{i} x_{i} \otimes s_{i} \in \mathscr{L}$ and $r \in I$. Then $\epsilon(r)=0$, since $I=P \cap R \subseteq P=\operatorname{ker} \epsilon$. Hence

$$
\operatorname{ev}_{P}(r z)=\sum x_{i} \epsilon\left(r s_{i}\right)=\sum x_{i} \epsilon(r) \epsilon\left(s_{i}\right)=0
$$

\footnotetext{
${ }^{4}$ Recall that there is no difference in the concept of maximal ideal if we view $\mathscr{L}$ as an $R$ - or $k$-Lie algebra.

${ }^{5} S$ is of finite type over $R$ and $R$ is assumed to be of finite type over $k$. Thus $S$ is of finite type over $k$ and therefore $S / P \simeq k$ by the Nullstellensatz.
} 
so $I \mathscr{L} \subseteq \operatorname{kerev}_{P}$. Since $\mathscr{I}=I \mathscr{L}$ is a maximal ideal and $\mathrm{ev}_{P}$ is nonzero, the kernel of $\mathrm{ev}_{P}$ is precisely $\mathscr{I}$.

We have now shown that $\mathscr{L} / \operatorname{ker} \phi$ is isomorphic to a direct sum of finitely many copies of $\mathfrak{g}$. Explicitly, $\operatorname{ker} \phi$ is the intersection of a (finite) family of distinct maximal ideals $M_{1}, \ldots, M_{n}$ in $\mathscr{L}$. Let $I_{1}, \ldots, I_{n}$ be the (distinct) maximal ideals of $R$ given by Theorem 2.9. For any collection $\underline{M}$ of maximal ideals $M_{1}, \ldots, M_{n}$ of $S$ lying over $I_{1}, \ldots, I_{n}$, respectively, the map

$$
\begin{aligned}
\mathrm{ev}_{\underline{M}}=\left(\mathrm{ev}_{M_{1}}, \ldots, \mathrm{ev}_{M_{n}}\right): \mathscr{L} & \rightarrow \mathfrak{g} \oplus \cdots \oplus \mathfrak{g}, \\
z & \mapsto\left(\operatorname{ev}_{M_{1}}(z), \ldots, \mathrm{ev}_{M_{n}}(z)\right)
\end{aligned}
$$

descends to an isomorphism $\operatorname{ev}_{\underline{M}}: \mathscr{L} / \operatorname{ker} \phi \rightarrow \mathfrak{g} \oplus \cdots \oplus \mathfrak{g}$.

Since the irreducible representations of $\mathfrak{g}^{\oplus n}=\mathfrak{g} \oplus \cdots \oplus \mathfrak{g}$ are precisely the tensor products

$$
\begin{aligned}
\rho=\left(\rho_{1}, \ldots, \rho_{n}\right): \mathfrak{g} \oplus \cdots \oplus \mathfrak{g} & \rightarrow \operatorname{End}_{k}\left(V_{1} \otimes \cdots \otimes V_{n}\right), \\
\left(x_{1}, \ldots, x_{n}\right) & \mapsto \sum_{i=1}^{n} \mathrm{id} \otimes \cdots \otimes \rho_{i}\left(x_{i}\right) \otimes \cdots \otimes \mathrm{id}
\end{aligned}
$$

of simple $\mathfrak{g}$-modules $\left(\rho_{i}, V_{i}\right)$, we now have a complete list of the simple $\mathscr{L}$-modules.

Theorem 3.4. Let $\phi: \mathscr{L} \rightarrow$ End $_{k}(V)$ be a finite-dimensional irreducible representation of $\mathscr{L}$. Then there exists a finite collection $\underline{P}=\left(P_{1}, \ldots, P_{n}\right)$ of maximal ideals of $S$ with $P_{i} \cap R \neq P_{j} \cap R$ for $i \neq j$, and a simple $\mathfrak{g}^{\oplus n}$-module $\left(\rho, V_{1} \otimes \cdots \otimes V_{n}\right)$ such that $V \simeq V_{1} \otimes \cdots \otimes V_{n}$ and $\phi=\rho \circ \mathrm{ev}_{\underline{P}}$.

Remark 3.5. The converse of Theorem 3.4 is obvious. Given a collection of maximal ideals $P_{1}, \ldots, P_{n}$ of $S$ for which the ideals $P_{i} \cap R$ of $R$ are pairwise distinct, the Chinese remainder theorem gives an isomorphism

$$
\mathscr{L} / M_{1} \oplus \cdots \oplus \mathscr{L} / M_{n} \simeq \mathscr{L} / \cap_{i} M_{i}
$$

where $\mathcal{M}_{i}=\left(P_{i} \cap R\right) \mathscr{L}$. (This uses the fact that the $P_{i} \cap R$ are maximal, as shown in the proof of Theorem 2.9.) Thus the map

$$
\mathscr{L} \rightarrow \mathscr{L} / M_{1} \oplus \cdots \oplus \mathscr{L} / M_{n} \simeq \mathfrak{g}^{\oplus n}
$$

is surjective, so the pullback of any simple $\mathfrak{g}^{\oplus n}$-module $V=V_{1} \otimes \cdots \otimes V_{n}$ will be a simple $\mathscr{L}$-module.

3b. Isomorphism classes of simple modules. Fix a Cartan subalgebra $\mathfrak{h}$ of $\mathfrak{g}$ and an épinglage of $(\mathfrak{g}, \mathfrak{h})$; see [Bourbaki 1975, VIII, §4.1]. Given a maximal ideal $M \in \operatorname{Max}(S)$ and a finite-dimensional representation $\rho: \mathfrak{g} \rightarrow \operatorname{End}_{k}(W)$, we write 
$W(M)$ for the vector space $W$, viewed as an $\mathscr{L}$-module with action given by the composition of maps

$$
\mathscr{L} \hookrightarrow \mathfrak{g} \otimes S \stackrel{\mathrm{ev} M}{\longrightarrow} \mathfrak{g} \stackrel{\rho}{\rightarrow} \operatorname{End}_{k}(W)
$$

where $\mathrm{ev}_{M}$ is the quotient map

$$
\begin{aligned}
\mathrm{ev}_{M}: \mathfrak{g} \otimes S & \rightarrow(\mathfrak{g} \otimes S) /(\mathfrak{g} \otimes M)=\mathfrak{g} \otimes(S / M) \simeq \mathfrak{g}, \\
x \otimes s & \mapsto(x \otimes s)(M)=s(M) x
\end{aligned}
$$

for all $x \in \mathfrak{g}$ and $s \in S$. For each automorphism $\alpha \in \operatorname{Aut}_{S \text {-Lie }}(\mathfrak{g}(S))$ and $M \in \operatorname{Max}(S)$, we write $\alpha(M) \in \operatorname{Aut}(\mathfrak{g})$ for the automorphism defined by

$$
(\alpha(M))(x)=(\alpha(x \otimes 1))(M)=\operatorname{ev}_{M}(\alpha(x \otimes 1))
$$

for each $x \in \mathfrak{g}$. It is straightforward to verify that the map

$$
\operatorname{Aut}_{S-\operatorname{Lie}}(\mathfrak{g}(S)) \rightarrow \operatorname{Aut}(\mathfrak{g}), \quad \alpha \mapsto \alpha(M)
$$

is a group homomorphism for each $M \in \operatorname{Max}(S)$. We write Out $\alpha(M)$ and $\operatorname{Int} \alpha(M)$ for the outer and inner parts, respectively, of the automorphism

$$
\alpha(M)=\operatorname{Int} \alpha(M) \circ \text { Out } \alpha(M) .
$$

See [Bourbaki 1975, VIII, $\$ 5.3$ corollaire 1] for details.

By Theorem 3.4, the (finite-dimensional) simple $\mathscr{L}$-modules are those of the form $V(\lambda, M)=V_{\lambda_{1}}\left(M_{1}\right) \otimes \cdots \otimes V_{\lambda_{n}}\left(M_{n}\right)$, where each $\lambda_{i}$ is in the set $P_{+}^{\times}$of nonzero dominant integral weights, $V_{\lambda_{i}}$ is the simple $\mathfrak{g}$-module of highest weight $\lambda_{i}$, and $M=\left(M_{1}, \ldots, M_{n}\right)$ is an $n$-tuple of maximal ideals of $S$ lying over distinct (closed) points of $\operatorname{Spec}(R)$.

Lemma 3.6. Suppose that the $\mathscr{L}$-modules $V(\lambda, M)=V_{\lambda_{1}}\left(M_{1}\right) \otimes \cdots \otimes V_{\lambda_{m}}\left(M_{m}\right)$ and $V(\mu, N)=V_{\mu_{1}}\left(N_{1}\right) \otimes \cdots \otimes V_{\mu_{n}}\left(N_{n}\right)$ are isomorphic for certain $\lambda_{1}, \ldots, \lambda_{m}$, $\mu_{1}, \ldots, \mu_{n} \in P_{+}^{\times}$and $M_{1}, \ldots, M_{m}, N_{1}, \ldots, N_{n} \in \operatorname{Max}(S)$. Then $m=n$, and up to reordering, $M_{i} \cap R=N_{i} \cap R$ for all $i$.

Proof. Let $\phi_{\lambda, M}: \mathscr{L} \rightarrow \operatorname{End}_{k}(V(\lambda, M))$ and $\phi_{\mu, N}: \mathscr{L} \rightarrow \operatorname{End}_{k}(V(\mu, N))$ be the homomorphisms determining the module actions. Since $V(\lambda, M) \simeq V(\mu, N)$, their kernels are equal, so

$$
\bigcap_{i=1}^{m}\left(M_{i} \cap R\right) \mathscr{L}=\operatorname{ker} \phi_{\lambda, M}=\operatorname{ker} \phi_{\mu, N}=\bigcap_{j=1}^{n}\left(N_{j} \cap R\right) \mathscr{L} .
$$


By Lemma 2.1(2) and Lemma 2.6,

$$
\begin{aligned}
\bigcap_{i=1}^{m}\left(M_{i} \cap R\right) & =\left(\bigcap_{i=1}^{m}\left(M_{i} \cap R\right) S\right) \cap R=J\left(\bigcap_{i=1}^{m}\left(M_{i} \cap R\right) \mathscr{L}\right) \cap R \\
& =J\left(\bigcap_{j=1}^{n}\left(N_{j} \cap R\right) \mathscr{L}\right) \cap R=\bigcap_{j=1}^{n}\left(N_{j} \cap R\right) .
\end{aligned}
$$

For $I \subseteq R$, let $\operatorname{Var} I$ be the set of $\mathfrak{m} \in \operatorname{Spec} R$ with $I \subseteq \mathfrak{m}$. Then

$$
\begin{aligned}
\bigcup_{i=1}^{m}\left\{M_{i} \cap R\right\} & =\bigcup_{i=1}^{m} \operatorname{Var}\left(M_{i} \cap R\right)=\operatorname{Var}\left(\bigcap_{i=1}^{m}\left(M_{i} \cap R\right)\right) \\
& =\operatorname{Var}\left(\bigcap_{j=1}^{n}\left(N_{j} \cap R\right)\right)=\bigcup_{j=1}^{n}\left\{N_{j} \cap R\right\} .
\end{aligned}
$$

Thus $m=n$, and after reordering, $M_{i} \cap R=N_{i} \cap R$ for all $i$.

Recall that $u_{\gamma}$ is the image of $\gamma \in \Gamma=\mathscr{G}_{a l}(S / R)$ under the Galois cocycle $u: \Gamma \rightarrow \operatorname{Aut}_{S \text {-Lie }}(\mathfrak{g}(S))$. The group $\Gamma$ acts on the set of pairs $(\lambda, M) \in P_{+}^{\times} \times \operatorname{Max}(S)$ by ${ }^{\gamma}(\mu, N)=\left(\mu \circ\right.$ Out $\left.u_{\gamma}{ }^{-1}\left({ }^{\gamma} N\right),{ }^{\gamma} N\right)$.

\section{Proposition 3.7. Suppose}

$V(\lambda, M)=V_{\lambda_{1}}\left(M_{1}\right) \otimes \cdots \otimes V_{\lambda_{n}}\left(M_{n}\right) \quad$ and $\quad V(\mu, N)=V_{\mu_{1}}\left(N_{1}\right) \otimes \cdots \otimes V_{\mu_{n}}\left(N_{n}\right)$

are irreducible $\mathscr{L}$-modules with $\lambda, \mu \in\left(P_{+}^{\times}\right)^{n}$ and $M_{i} \cap R=N_{i} \cap R$ for all $i$. Then $V(\lambda, M) \simeq V(\mu, N)$ if and only if there exist $\gamma_{1}, \ldots, \gamma_{n} \in \Gamma$ such that

$$
\left(\lambda_{i}, M_{i}\right)={ }^{\gamma_{i}}\left(\mu_{i}, N_{i}\right) \text { for } i=1, \ldots, n .
$$

Proof. Let $\phi_{\lambda, M}: \mathscr{L} \rightarrow \operatorname{End}_{k}(V(\lambda, M))$ and $\phi_{\mu, N}: \mathscr{L} \rightarrow \operatorname{End}_{k}(V(\mu, N))$ be the homomorphisms defining the module actions. Since each $\lambda_{i}$ is nonzero, the kernel of the action of $\mathfrak{g}^{\oplus n}$ on $V(\lambda, M)$ is trivial, and the evaluation maps $\mathrm{ev}_{M_{i}}$ induce an automorphism

$$
\mathrm{ev}_{M}=\mathrm{ev}_{M_{1}} \oplus \cdots \oplus \mathrm{ev}_{M_{n}}: \mathscr{L} / \operatorname{ker} \phi_{\lambda, M} \stackrel{\sim}{\longrightarrow} \mathfrak{g}^{\oplus n} .
$$

Similarly, ev $\mathrm{ev}_{N}: \mathscr{L} / \operatorname{ker} \phi_{\mu, N} \rightarrow \mathfrak{g}^{\oplus n}$ is a Lie algebra isomorphism.

Let $\mathfrak{g}=\mathfrak{n}_{-} \oplus \mathfrak{h} \oplus \mathfrak{n}_{+}$be the triangular decomposition of $\mathfrak{g}$ relative to the épinglage of $(\mathfrak{g}, \mathfrak{h})$. We pull back the corresponding triangular decomposition of $\mathfrak{g}^{\oplus n}$ to obtain the triangular decomposition

$$
\mathscr{L} / \operatorname{ker} \phi_{\lambda, M}=\mathrm{ev}_{M}^{-1}\left(\mathfrak{n}_{-}^{\oplus n}\right) \oplus \mathrm{ev}_{M}^{-1}\left(\mathfrak{h}^{\oplus n}\right) \oplus \mathrm{ev}_{M}^{-1}\left(\mathfrak{n}_{+}^{\oplus n}\right) .
$$

The representations $V(\lambda, M)$ and $V(\mu, N)$ will be isomorphic precisely when they have the same highest weights relative to the decomposition (3.8). 
The Galois group $\Gamma=\operatorname{Gal}(S / R)$ acts transitively on the fibers of the pullback map $\operatorname{Spec}(S) \rightarrow \operatorname{Spec}(R)$ over maximal ideals of $R$. Choose $\gamma_{i} \in \Gamma$ so that $M_{i}={ }^{\gamma_{i}} N_{i}$ for all $i$.

Let $\mathfrak{g}^{i}=0 \oplus \cdots \oplus \mathfrak{g} \oplus \cdots \oplus 0$ be the $i$-th component of $\mathfrak{g}^{\oplus n}$. Note that

$$
\operatorname{ev}_{M}^{-1}\left(\mathfrak{g}^{i}\right)=\bigcap_{r \neq i} \operatorname{kerev}_{M_{r}}=\bigcap_{r \neq i}\left(M_{r} \cap R\right) \mathscr{L}=\bigcap_{r \neq i}\left(N_{r} \cap R\right) \mathscr{L}=\bigcap_{r \neq i} \operatorname{kerev}_{N_{r}} .
$$

Therefore, $\operatorname{ev}_{N_{j}} \circ \mathrm{ev}_{M}^{-1}\left(\mathfrak{g}^{i}\right)=0$ for all $i \neq j$, and

$$
\operatorname{ev}_{N} \circ \operatorname{ev}_{M}^{-1}\left(x^{i}\right)=\iota_{i} \circ \operatorname{ev}_{N_{i}} \circ \operatorname{ev}_{M}^{-1}\left(x^{i}\right)=\iota_{i} \circ \operatorname{ev}_{N_{i}} \circ \operatorname{ev}_{M_{i}}^{-1}(x)
$$

for all $x^{i} \in \mathfrak{g}^{i}$, where $\iota_{i}$ is the inclusion of $\mathfrak{g}$ as the $i$-th component of $\mathfrak{g}^{\oplus n}$ :

$$
\iota_{i}: \mathfrak{g} \hookrightarrow 0 \oplus \cdots \oplus \mathfrak{g} \oplus \cdots \oplus 0 \subseteq \mathfrak{g}^{\oplus n} .
$$

Relative to the decomposition (3.8), the highest weight of $V(\lambda, M)$ is thus $\sum_{i=1}^{n} \lambda_{i} \circ \mathrm{ev}_{M_{i}}$ and the highest weight of $V(\mu, N)$ is $\sum_{i=1}^{n} v_{i} \circ \mathrm{ev}_{N_{i}}$, where $v_{i} \in$ $\left(\mathrm{ev}_{N_{i}} \circ \mathrm{ev}_{M_{i}}^{-1}(\mathfrak{h})\right)^{*}$ is the highest weight of $V_{\mu_{i}}$, relative to the new triangular decomposition

$$
\mathfrak{g}=\operatorname{ev}_{N_{i}} \circ \operatorname{ev}_{M_{i}}^{-1}\left(\mathfrak{n}_{-}\right) \oplus \operatorname{ev}_{N_{i}} \circ \operatorname{ev}_{M_{i}}^{-1}(\mathfrak{h}) \oplus \operatorname{ev}_{N_{i}} \circ \operatorname{ev}_{M_{i}}^{-1}\left(\mathfrak{n}_{+}\right)
$$

By [Lau 2010, Lemma 5.2], $v_{i}=\mu_{i} \circ \tau_{i}^{-1}$, where $\tau_{i}=\operatorname{Int}\left(\mathrm{ev}_{N_{i}} \circ \mathrm{ev}_{M_{i}}^{-1}\right)$. That is, $V(\lambda, M) \simeq V(\mu, N)$ if and only if

$$
\sum_{i=1}^{n} \lambda_{i} \circ \operatorname{ev}_{M_{i}}=\sum_{i=1}^{n} \mu_{i} \circ \tau_{i}^{-1} \circ \operatorname{ev}_{N_{i}}
$$

on $\mathrm{ev}_{M}^{-1}\left(\mathfrak{h}^{\oplus n}\right)$. For the $i$-th component $\mathfrak{h}^{i}=0 \oplus \cdots \oplus \mathfrak{h} \oplus \cdots \oplus 0$, we have

$$
\mathrm{ev}_{M}^{-1}\left(\mathfrak{h}^{i}\right) \subseteq \operatorname{ev}_{M}^{-1}\left(\mathfrak{g}^{i}\right)=\bigcap_{j \neq i}\left(M_{j} \cap R\right) \mathscr{L}
$$

so $\lambda_{j} \circ \operatorname{ev}_{M_{j}}\left(\operatorname{ev}_{M}^{-1}\left(\mathfrak{h}^{i}\right)\right)=0$ for $i \neq j$. Therefore, $V(\lambda, M) \simeq V(\mu, N)$ if and only if $\lambda_{i} \circ \mathrm{ev}_{M_{i}}=\mu_{i} \circ \tau_{i}^{-1} \circ \mathrm{ev}_{N_{i}}$ for all $i$; that is, if and only if $\lambda_{i}=\mu_{i} \circ \operatorname{Out}\left(\mathrm{ev}_{N_{i}} \circ \mathrm{ev}_{M_{i}}^{-1}\right)$.

We now simplify the expression for the automorphism $\mathrm{ev}_{N_{i}} \circ \mathrm{ov}_{M_{i}}^{-1}: \mathfrak{g} \rightarrow \mathfrak{g}$. For $x \in \mathfrak{g}$, write $\mathrm{ev}_{M_{i}}^{-1}(x)=\sum_{j} x_{j} \otimes s_{j}+\operatorname{kerev}_{M_{i}} \in \mathscr{L} / \operatorname{kerev}_{M_{i}}=\mathscr{L} / \operatorname{ker}_{N_{i}}$, where $x_{j} \in \mathfrak{g}$ and $s_{j} \in S$ for all $j$. Then $\mathrm{ev}_{N_{i}} \circ \mathrm{ev}_{M_{i}}^{-1}(x)=\sum_{j} s_{j}\left(N_{i}\right) x_{j}$. By definition,

$$
s_{j}\left(N_{i}\right)+N_{i}=s_{j}+N_{i} \in S / N_{i},
$$

and $s_{j}\left(N_{i}\right) \in k \subseteq R$ is clearly fixed by $\gamma_{i} \in \Gamma$. Hence

$$
s_{j}\left(N_{i}\right)+{ }^{\gamma_{i}} N_{i}={ }^{\gamma_{i}} s_{j}+{ }^{\gamma_{i}} N_{i} \in S /{ }^{\gamma_{i}} N_{i}=S / M_{i},
$$


and $s_{j}\left(N_{i}\right)={ }^{\gamma_{i}} s_{j}\left(M_{i}\right)$. Therefore,

$$
\operatorname{ev}_{N_{i}} \circ \operatorname{ev}_{M_{i}}^{-1}(x)=\sum_{j}{ }^{\gamma_{i}} s_{j}\left(M_{i}\right) x_{j}
$$

Moreover, $\sum_{j} x_{j} \otimes s_{j} \in \mathscr{L}=\left\{z \in \mathfrak{g} \otimes S \mid u_{\gamma}{ }^{\gamma} z=z\right.$ for all $\left.\gamma \in \Gamma\right\}$, so

$$
\begin{aligned}
\operatorname{ev}_{N_{i}} \circ \operatorname{ev}_{M_{i}}^{-1}(x) & ={ }^{\gamma_{i}}\left(\sum_{j} x_{j} \otimes s_{j}\right)\left(M_{i}\right)=\left(u_{\gamma_{i}}\right)^{-1}\left(\sum_{j} x_{j} \otimes s_{j}\right)\left(M_{i}\right) \\
& =u_{\gamma_{i}}^{-1}\left(M_{i}\right) \sum_{j} s_{j}\left(M_{i}\right) x_{j}=u_{\gamma_{i}}^{-1}\left(M_{i}\right)(x)
\end{aligned}
$$

and $\operatorname{ev}_{N_{i}} \circ \mathrm{ev}_{M_{i}}^{-1}=u_{\gamma_{i}}^{-1}\left(M_{i}\right)$. Hence $V(\lambda, M) \simeq V(\mu, N)$ if and only if there exist $\gamma_{1}, \ldots, \gamma_{n} \in \Gamma$ such that $\gamma_{i}\left(\mu_{i}, N_{i}\right)=\left(\lambda_{i}, M_{i}\right)$ for all $i$.

We identify the $\mathscr{L}$-module $V(\lambda, M)=V_{\lambda_{1}}\left(M_{1}\right) \otimes \cdots \otimes V_{\lambda_{n}}\left(M_{n}\right)$ with the map

$$
\chi_{[\lambda, M]}: \operatorname{Max}(S) \rightarrow P_{+},
$$

where $\chi_{[\lambda, M]}=\sum_{\gamma \in \Gamma} \sum_{i=1}^{n} \chi_{\gamma_{\left(\lambda_{i}, M_{i}\right)}}$ and

$$
\chi_{\left(\mu_{i}, N_{i}\right)}: \operatorname{Max}(S) \rightarrow P_{+}, \quad I \mapsto \begin{cases}\mu_{i} & \text { if } I=N_{i}, \\ 0 & \text { otherwise. }\end{cases}
$$

The Galois group $\Gamma$ acts on the set $\mathscr{F}$ of finitely supported functions $\operatorname{Max}(S) \rightarrow P_{+}$, by identifying each function $f$ with the set of ordered pairs $\{(f(M), M) \mid M \in$ $\operatorname{Max}(S)\}$ and defining $\gamma_{f}=\{\gamma(f(M), M) \mid M \in \operatorname{Max}(S)\}$. The function $\chi_{[\gamma, M]}$ is then $\Gamma$-invariant, and the set $\mathscr{F}^{\Gamma}$ of $\Gamma$-invariant functions in $\mathscr{F}$ is in bijection with the set $\mathscr{C}$ of isomorphism classes [ $V$ ] of (finite-dimensional) simple $\mathscr{L}$-modules $V$ :

Theorem 3.9. The map $\psi:[V(\lambda, M)] \mapsto \chi_{[\lambda, M]}$ is a well-defined natural bijection between $\mathscr{C}$ and $\mathscr{F} \Gamma$.

Proof. By Theorem 3.4, Lemma 3.6, and Proposition 3.7, two simple $\mathscr{L}$-modules $W_{1}$ and $W_{2}$ are isomorphic if and only if there exist $n \geq 0$, ordered pairs

$$
(M, \lambda),(N, \mu) \in(\operatorname{Max}(S))^{n} \times\left(P_{+}^{\times}\right)^{n}
$$

with $M_{i} \cap R=N_{i} \cap R \neq N_{j} \cap R=M_{j} \cap R$ for $i \neq j$, and $\gamma_{1}, \ldots, \gamma_{n} \in \Gamma$ such that $W_{1} \simeq V(\lambda, M), W_{2} \simeq V(\mu, N)$, and $\left(M_{i}, \lambda_{i}\right)=\gamma_{i}\left(N_{i}, \mu_{i}\right)$ for all $i$. Thus $V(\lambda, M) \simeq V(\mu, N)$ if and only if $\chi_{[\lambda, M]}=\chi_{[\mu, N]}$. In particular, the map $\psi: \mathscr{C} \rightarrow \mathscr{F}^{\Gamma}$ is well-defined and injective. It is also surjective, as the support of any $f \in \mathscr{F}^{\Gamma}$ decomposes into a disjoint union of $\Gamma$-orbits. Therefore,

$$
f=\sum_{\gamma \in \Gamma} \sum_{i=1}^{m} \chi_{\gamma\left(\lambda_{i}, M_{i}\right)}
$$


for some collection of orbit representatives $M_{1}, \ldots, M_{m} \in \operatorname{Max}(S)$.

\section{Applications}

In this section, $k$ will denote an algebraically closed field of characteristic zero.

4a. Multiloop algebras. Multiloop algebras are multivariable generalizations of the loop algebras in affine Kac-Moody theory. The study of these algebras and their extensions includes a substantial body of work on (twisted and untwisted) multiloop, toroidal, and extended affine Lie algebras. The representation theory of multiloop algebras has also been adapted to include generalized current algebras and equivariant map algebras [Chari et al. 2010; Neher et al. 2012]. When $R$ and $S$ are Laurent polynomial rings, the intersection of the class of algebras with the class of twisted forms discussed in the present paper includes multiloop algebras (Section 4a), but not Margaux algebras (Section 4b), for instance.

Let $\mathfrak{g}$ be a finite-dimensional simple Lie algebra over $k$, with commuting automorphisms $\sigma_{1}, \ldots, \sigma_{N}: \mathfrak{g} \rightarrow \mathfrak{g}$ of finite orders $m_{1}, \ldots, m_{N}$, respectively. Fix a primitive $m_{j}$-th root of unity $\xi_{j} \in k$ for each $j$, and let $R=k\left[t_{1}^{ \pm m_{1}}, \ldots, t_{N}^{ \pm m_{N}}\right] \subseteq$ $S=k\left[t_{1}^{ \pm 1}, \ldots, t_{N}^{ \pm 1}\right]$.

The (twisted) multiloop algebra $\mathscr{L}=\mathscr{L}(\mathfrak{g}, \sigma)$ is a $\mathbb{Z}^{N}$-graded subalgebra of $\mathfrak{g}(S)=\mathfrak{g} \otimes S:$

$$
\mathscr{L}(\mathfrak{g}, \sigma)=\bigoplus_{j \in \mathbb{Z}^{N}} \mathfrak{g}_{j} \otimes t^{j},
$$

where $j=\left(j_{1}, \ldots, j_{N}\right), \mathfrak{g}_{j}=\left\{x \in \mathfrak{g} \mid \sigma_{i}(x)=\xi_{i}^{j_{i}} x\right.$ for $\left.i=1, \ldots, N\right\}$, and $t^{j}=t_{1}^{j_{1}} t_{2}^{j_{2}} \cdots t_{N}^{j_{N}}$. It is easy to see that $\mathscr{L}$ is a Lie algebra over $R$ and an $S / R$ form of $\mathfrak{g}(R)$.

Specializing our main theorems to the case of multiloop algebras, we recover the results of [Lau 2010]. Maximal ideals $M_{i}=M_{a_{i}}=\left(t_{1}-a_{i 1}, \ldots, t_{N}-a_{i N}\right)$ of $S$ correspond to points $a_{i}=\left(a_{i 1}, \ldots, a_{i N}\right)$ on the algebraic $n$-torus $\left(k^{\times}\right)^{N}=k^{\times} \times$ $\cdots \times k^{\times}$. Note that $M_{i} \cap R$ is the ideal (of $R$ ) of polynomials vanishing at $a_{i}$. Thus $M_{i} \cap R \in \operatorname{Max} R$ is generated by $\left\{t_{1}^{m_{1}}-a_{i 1}^{m_{1}}, \ldots, t_{N}^{m_{N}}-a_{i N}^{m_{N}}\right\}$. Therefore, $M_{i} \cap R=$ $M_{j} \cap R$ if and only if $m\left(a_{i}\right)=m\left(a_{j}\right)$, where we write $m\left(a_{\ell}\right)=\left(a_{\ell 1}^{m_{1}}, \ldots, a_{\ell N}^{m_{N}}\right)$ for all $a_{\ell} \in\left(k^{\times}\right)^{N}$.

The Galois group $\Gamma=\mathscr{G a l}_{\text {al }}(S / R)$ is $\mathbb{Z}_{m_{1}} \times \cdots \times \mathbb{Z}_{m_{N}}$, where each $\mathbb{Z}_{m_{i}}$ is generated by an element

$$
\alpha_{i}: t_{j} \mapsto \begin{cases}\xi_{i} t_{i} & \text { if } i=j, \\ t_{j} & \text { otherwise. }\end{cases}
$$

The 1-cocycle $u: \Gamma \rightarrow \operatorname{Aut}_{S \text {-Lie }}(\mathfrak{g}(S))$ corresponding to $\mathscr{L}$ is given by

$$
u_{\gamma}=\sigma_{1}^{-r_{1}} \cdots \sigma_{N}^{-r_{N}} \otimes 1,
$$


for each $\gamma=\left(\alpha_{1}^{r_{1}}, \ldots, \alpha_{N}^{r_{N}}\right) \in \Gamma$. Then $u_{\gamma}(M)=\sigma_{1}^{-r_{1}} \cdots \sigma_{N}^{-r_{N}}$ for all $M \in \operatorname{Max}(S)$. The fact that

$$
u_{\gamma}: \operatorname{Max}(S) \rightarrow \text { Aut } \mathfrak{g}, \quad M \mapsto u_{\gamma}(M)
$$

is constant means that the action of $\Gamma$ on $P_{+}^{\times} \times \operatorname{Max}(S)$ splits into separate actions of $\Gamma$ on $\operatorname{Max}(S)$ and on $P_{+}^{\times}$by

$$
\psi: \Gamma \times P_{+}^{\times} \rightarrow P_{+}^{\times}, \quad(\gamma, \lambda) \mapsto \lambda \circ \text { Out } \sigma_{1}^{-r_{1}} \cdots \sigma_{N}^{-r_{N}} .
$$

In this language, $\Gamma$ acts on $P_{+}^{\times} \times \operatorname{Max}(S)$ as $\gamma(\lambda, M)=\left(\psi\left(\gamma^{-1}, \lambda\right),{ }^{\gamma} M\right)$. The $\Gamma$-invariant functions $\chi_{[\lambda, M]}: \operatorname{Max}(S) \rightarrow P_{+}$become $\Gamma$-equivariant functions under the new action $\psi$ on $P_{+}^{\times}$. We thus recover the following theorem [Lau 2010, Corollary 4.4, Theorem 4.5, and Corollary 5.10]:

Theorem 4.1. (1) The finite-dimensional simple modules of $\mathscr{L}(\mathfrak{g} ; \sigma)$ are those of the form $V(\lambda, a)=V_{\lambda_{1}}\left(M_{a_{1}}\right) \otimes \cdots \otimes V_{\lambda_{n}}\left(M_{a_{n}}\right)$ for $n \geq 0, a_{i} \in\left(k^{\times}\right)^{N}$, and $m\left(a_{i}\right) \neq m\left(a_{j}\right)$ whenever $i \neq j$.

(2) The isomorphism classes of finite-dimensional simple $\mathscr{L}(\mathfrak{g} ; \sigma)$-modules are in bijection with the finitely supported $\Gamma$-equivariant maps $\left(k^{\times}\right)^{N} \rightarrow P_{+}$.

4b. Azumaya and Margaux algebras. Fix Laurent polynomial rings

$$
R=k\left[t_{1}^{ \pm 2}, t_{2}^{ \pm 2}\right] \quad \text { and } \quad S=k\left[t_{1}^{ \pm 1}, t_{2}^{ \pm 1}\right] .
$$

Let $A=A(1,2)$ be the standard Azumaya algebra, the unital associative $R$-algebra generated by $\left\{T_{1}^{ \pm 1}, T_{2}^{ \pm 1}\right\}$ with relations $T_{2} T_{1}=-T_{1} T_{2}$ and $T_{i}^{2}=t_{i}^{2}$ for $i=1,2$. Then $A$ is an $S / R$-form of the associative algebra $M_{2}(R)$ of $2 \times 2$ matrices over $R$, as can be readily verified using one of the well-known representations of the quaternions as matrices in $M_{2}(\mathbb{C})$.

Since $\mathbf{P G L} \mathbf{L}_{2}$ is the automorphism group (scheme) of both $M_{2}(k)$ and $\mathfrak{s l}_{2}(k)$, there is a natural correspondence between $S / R$-forms of $M_{2}(R)$ and $\mathfrak{s l}_{2}(R)$. Namely, given any $S / R$-form $B$ of the matrix algebra $M_{2}(R)$, view $B$ as a Lie algebra Lie $B$ with bracket $[a, b]=a b-b a$. Its derived subalgebra $(\operatorname{Lie} B)^{\prime}=\operatorname{Span}\{[a, b] \mid$ $a, b \in B\}$ is then an $S / R$-form of $\mathfrak{s l}_{2}(R)$.

Applying this construction to $\mathscr{L}_{1}=(\text { Lie } A)^{\prime}$ and computing explicitly, it follows that $\mathscr{L}_{1} \simeq \mathscr{L}\left(\mathfrak{s l}_{2}(k), \sigma_{1}, \sigma_{2}\right)$ where $\sigma_{1}$ and $\sigma_{2}$ are conjugation by $\left(\begin{array}{cc}1 & 0 \\ 0 & -1\end{array}\right)$ and $\left(\begin{array}{ll}0 & 1 \\ 1 & 0\end{array}\right)$, respectively [Gille and Pianzola 2007]. Therefore, we obtain the representations of $\mathscr{L}_{1}$ as in the previous section.

Surprisingly, not every twisted form of $\mathfrak{g}\left(k\left[t_{1}^{ \pm 1}, t_{2}^{ \pm 1}\right]\right)$ is a multiloop algebra. This can be seen using loop torsors. The only known $S / R$-forms of $\mathfrak{g}(R)$ that are not isomorphic to multiloop algebras are called Margaux algebras. The simplest of these can be constructed concretely as follows. See [Gille and Pianzola 2007] for details. 
Let $A, R$, and $S$ be as in Section $4 \mathrm{a}$. The right $A$-module

$$
M=\left\{(\lambda, \mu) \in A \oplus A \mid\left(1+T_{1}\right) \lambda=\left(1+T_{2}\right) \mu\right\}
$$

is projective but not free. This can be used to show that its endomorphism ring $M=\operatorname{End}_{A}(M)$, while also an $S / R$-form of $M_{2}(R)$, is not isomorphic to $A$ as an $A$-algebra. It follows that $\mathscr{L}_{1}$ and $\mathscr{L}_{2}=(\operatorname{Lie} M)^{\prime}$ are nonisomorphic $S / R$-forms of $\mathfrak{s l}_{2}(R)$. By the classification of involutions in $\mathrm{PGL}_{2}(k)$ and a study of loop torsors, it can be shown that $\mathscr{L}_{2}$ is not a (twisted) multiloop algebra.

By Theorems 3.4 and 3.9, the irreducible representations of $\mathscr{L}_{2}$ are the tensor products $V(\lambda, M)=V_{\lambda_{1}}\left(M_{1}\right) \otimes \cdots \otimes V_{\lambda_{n}}\left(M_{n}\right)$, where $\lambda_{1}, \ldots, \lambda_{n} \in \mathbb{Z}_{+} \backslash\{0\}$ are highest weights of $\mathfrak{s l}_{2}(k)$ and $M_{i}=\left\langle t_{1}-a_{i 1}, t_{2}-a_{i 2}\right\rangle$ are maximal ideals of $S=k\left[t_{1}^{ \pm 1}, t_{2}^{ \pm 1}\right]$ corresponding to points in distinct fibers over Spec $R$. That is, $\left(a_{i 1}^{2}, a_{i 2}^{2}\right) \neq\left(a_{j 1}^{2}, a_{j 2}^{2}\right)$ for $i \neq j$.

Two such representations

$V(\lambda, M)=V_{\lambda_{1}}\left(M_{1}\right) \otimes \cdots \otimes V_{\lambda_{m}}\left(M_{m}\right) \quad$ and $\quad V(\mu, N)=V_{\mu_{1}}\left(N_{1}\right) \otimes \cdots \otimes V_{\mu_{n}}\left(N_{n}\right)$ are isomorphic precisely when the corresponding $\mathscr{G}_{a l}(S / R)$-invariant functions $\chi_{[\lambda, M]}$ and $\chi_{[\mu, N]}$ are equal. But the action

$$
\gamma_{\left(\lambda_{i}, M_{i}\right)}=\left(\lambda_{i} \circ \text { Out } u_{\gamma}{ }^{-1}\left({ }^{\gamma} M_{i}\right),{ }^{\gamma} M_{i}\right)
$$

is simply an action on $\operatorname{Max}(S)$,

$$
\left.\gamma_{(} \lambda_{i}, M_{i}\right)=\left(\lambda_{i},{ }^{\gamma} M_{i}\right)
$$

since $u_{\gamma}{ }^{-1}\left({ }^{\gamma} M\right) \in$ Aut $\mathfrak{s l}_{2}(k)$, and every automorphism of $\mathfrak{s l}_{2}(k)$ is inner! Thus $V(\lambda, M) \simeq V(\mu, N)$ if and only if (after reordering the tensor factors) $m=n$, $\lambda_{i}=\mu_{i}$, and the $a_{i}, b_{i} \in k^{\times} \times k^{\times}$corresponding to $M_{i}$ and $N_{i}$ satisfy $a_{i j}= \pm b_{i j}$ for all $i$ and $j$.

As for any Galois extension $S / R$, the isomorphism classes of the (finite-dimensional) simple modules of any $S / R$-form of $\mathfrak{s l}_{2}(R)$ are given by restrictions of the same evaluation modules of $\mathfrak{s l}_{2}(S)$. In particular, the irreducible $\mathscr{L}_{1}$ - and $\mathscr{L}_{2}$-modules come from the same $\mathfrak{s l}_{2}(S)$-modules.

\section{Acknowledgements}

We would like to thank Jean Auger and Zhihua Chang for their careful reading of the manuscript.

\section{References}

[Bourbaki 1964] N. Bourbaki, Éléments de mathématique: Algèbre commutative, Chapitre 5, Actualités Scientifiques et Industrielles 1308, Hermann, Paris, 1964. MR 33 \#2660 Zbl 0205.34302 
[Bourbaki 1975] N. Bourbaki, Éléments de mathématique: Groupes et algèbres de Lie, Chapitre 7 et 8 , Actualités Scientifiques et Industrielles 1364, Hermann, Paris, 1975. MR 56 \#12077 Zbl 0329.17002

[Chari et al. 2010] V. Chari, G. Fourier, and T. Khandai, "A categorical approach to Weyl modules", Transform. Groups 15:3 (2010), 517-549. MR 2011m:17014 Zbl 1245.17004

[Gille and Pianzola 2007] P. Gille and A. Pianzola, "Galois cohomology and forms of algebras over Laurent polynomial rings", Math. Ann. 338:2 (2007), 497-543. MR 2008b:20055 Zbl 1131.11070

[Knus and Ojanguren 1974] M.-A. Knus and M. Ojanguren, Théorie de la descente et algèbres d'Azumaya, Lecture Notes in Math. 389, Springer, Berlin, 1974. MR 54 \#5209 Zbl 0284.13002

[Lau 2010] M. Lau, "Representations of multiloop algebras", Pacific J. Math. 245:1 (2010), 167-184. MR 2011f:17041 Zbl 1237.17012

[Matsumura 1989] H. Matsumura, Commutative ring theory, 2nd ed., Cambridge Studies in Advanced Mathematics 8, Cambridge University Press, 1989. MR 90i:13001 Zbl 0666.13002

[Milne 1980] J. S. Milne, Étale cohomology, Princeton Mathematical Series 33, Princeton University Press, 1980. MR 81j:14002 Zbl 0433.14012

[Neher et al. 2012] E. Neher, A. Savage, and P. Senesi, "Irreducible finite-dimensional representations of equivariant map algebras", Trans. Amer. Math. Soc. 364:5 (2012), 2619-2646. MR 2888222 Zbl 06028848

[Senesi 2010] P. Senesi, "Finite-dimensional representation theory of loop algebras: a survey", pp. 263-283 in Quantum affine algebras, extended affine Lie algebras, and their applications, edited by Y. Gao et al., Contemp. Math. 506, Amer. Math. Soc., Providence, RI, 2010. MR 2011d:17040 Zbl 05901955

Communicated by Georgia Benkart

Received 2011-11-07 Accepted 2012-03-03

michael.lau@mat.ulaval.ca Département de mathématiques et de statistique, Université Laval, Pavillon Vachon (Local 1056), 1045 av. de la Médecine, Québec, QC G1V0A6, Canada

a.pianzola@gmail.com

Department of Mathematical and Statistical Sciences, University of Alberta, 632 Central Academic Building, Edmonton, AB T6G2G1, Canada

Centro de Altos Estudios en Ciencias Exactas, Avenida de Mayo 866, (1084), Buenos Aires, Argentina 


\section{Algebra \& Number Theory}

msp.org/ant

\section{EDITORS}

MANAGING EDITOR

Bjorn Poonen

Massachusetts Institute of Technology

Cambridge, USA

\author{
EDITORIAL BOARD CHAIR \\ David Eisenbud \\ University of California \\ Berkeley, USA
}

\section{BOARD OF EDITORS}

Georgia Benkart

Dave Benson

Richard E. Borcherds

John H. Coates

J-L. Colliot-Thélène

Brian D. Conrad

Hélène Esnault

Hubert Flenner

Edward Frenkel

Andrew Granville

Joseph Gubeladze

Ehud Hrushovski

Craig Huneke

Mikhail Kapranov

Yujiro Kawamata

János Kollár

Yuri Manin

Barry Mazur

Philippe Michel
University of Wisconsin, Madison, USA

University of Aberdeen, Scotland

University of California, Berkeley, USA

University of Cambridge, UK

CNRS, Université Paris-Sud, France

University of Michigan, USA

Freie Universität Berlin, Germany

Ruhr-Universität, Germany

University of California, Berkeley, USA

Université de Montréal, Canada

San Francisco State University, USA

Hebrew University, Israel

University of Virginia, USA

Yale University, USA

University of Tokyo, Japan

Princeton University, USA

Northwestern University, USA

Harvard University, USA

École Polytechnique Fédérale de Lausanne
Susan Montgomery

Shigefumi Mori

Raman Parimala

Jonathan Pila

Victor Reiner

Karl Rubin

Peter Sarnak

Joseph H. Silverman

Michael Singer

Vasudevan Srinivas

J. Toby Stafford

Bernd Sturmfels

Richard Taylor

Ravi Vakil

Michel van den Bergh

Marie-France Vignéras

Kei-Ichi Watanabe

Efim Zelmanov
University of Southern California, USA

RIMS, Kyoto University, Japan

Emory University, USA

University of Oxford, UK

University of Minnesota, USA

University of California, Irvine, USA

Princeton University, USA

Brown University, USA

North Carolina State University, USA

Tata Inst. of Fund. Research, India

University of Michigan, USA

University of California, Berkeley, USA

Harvard University, USA

Stanford University, USA

Hasselt University, Belgium

Université Paris VII, France

Nihon University, Japan

University of California, San Diego, USA

\section{PRODUCTION}

production@msp.org

Silvio Levy, Scientific Editor

See inside back cover or msp.org/ant for submission instructions.

The subscription price for 2013 is US \$200/year for the electronic version, and \$350/year ( $\$ 40$, if shipping outside the US) for print and electronic. Subscriptions, requests for back issues and changes of subscribers address should be sent to MSP.

Algebra \& Number Theory (ISSN 1944-7833 electronic, 1937-0652 printed) at Mathematical Sciences Publishers, 798 Evans Hall \#3840, c/o University of California, Berkeley, CA 94720-3840 is published continuously online. Periodical rate postage paid at Berkeley, CA 94704, and additional mailing offices.

ANT peer review and production are managed by EditFLOW ${ }^{\circledR}$ from Mathematical Sciences Publishers.

\section{PUBLISHED BY}

mathematical sciences publishers

nonprofit scientific publishing

http://msp.org/

(C) 2013 Mathematical Sciences Publishers 


\section{Algebra \& Number Theory}

$\begin{array}{lll}\text { Volume } 7 & \text { No. } 2 & 2013\end{array}$

The system of representations of the Weil-Deligne group associated to an abelian variety 243 RUTGER NOOT

Fourier-Jacobi coefficients of Eisenstein series on unitary groups

BEI ZHANG

The phase limit set of a variety

MoUnIR NisSE and FRANK SoTTILE

Base change behavior of the relative canonical sheaf related to higher dimensional moduli

Zsolt PATAKFALVI

Two ways to degenerate the Jacobian are the same

JESSE LEO KASS

Arithmetic motivic Poincaré series of toric varieties

Helena Cobo Pablos and Pedro Daniel González Pérez

Maximal ideals and representations of twisted forms of algebras

Michael LAU and ARTURo PiAnZOLA

Higher Chow groups of varieties with group action 